\title{
@ु \\ Submersion of potassium clusters in helium nanodroplets
}

\author{
Lukas An der Lan, ${ }^{1}$ Peter Bartl,${ }^{1}$ Christian Leidlmair, ${ }^{1}$ Harald Schöbel, ${ }^{1}$ Stephan Denifl, ${ }^{1, *}$ Tilmann D. Märk, ${ }^{1}$ \\ Andrew M. Ellis, ${ }^{2, *}$ and Paul Scheier ${ }^{1}$ \\ ${ }^{1}$ Institut für Ionenphysik und Angewandte Physik and Research Platform Advanced Materials, Universität Innsbruck, \\ Technikerstr. 25, A-6020 Innsbruck, Austria \\ ${ }^{2}$ Department of Chemistry, University of Leicester, University Road, Leicester LE1 7RH, United Kingdom
}

(Received 23 January 2012; published 12 March 2012)

\begin{abstract}
Small alkali clusters do not submerge in liquid helium nanodroplets but instead survive predominantly in high spin states that reside on the surface of the nanodroplet. However, a recent theoretical prediction by Stark and Kresin [Phys. Rev. B 81, 085401 (2010)], based on a classical description of the energetics of bubble formation for a fully submerged alkali cluster, suggests that the alkali clusters can submerge on energetic grounds when they exceed a critical size. Following recent work on sodium clusters, where ion yield data from electron impact mass spectrometry was used to obtain the first experimental evidence for alkali cluster submersion, we report here on similar experiments for potassium clusters. Evidence is presented for full cluster submersion at $n>80$ for $\mathrm{K}_{n}$ clusters, which is in good agreement with the recent theoretical prediction. In an additional observation, we report "magic number" sizes for both $\mathrm{K}_{n}{ }^{+}$and $\mathrm{K}_{n}{ }^{2+}$ ions derived from helium droplets, which are found to be consistent with the jellium model.
\end{abstract}

DOI: 10.1103/PhysRevB.85.115414

PACS number(s): 68.08.Bc, 36.40.-c

\section{INTRODUCTION}

Most atoms and molecules that collide and attach to helium nanodroplets ultimately make their way inside the droplets. An interior equilibrium location is favored by the attractive forces between the dopant and the helium that, while generally very weak, still yield a lower energy scenario than a surface location. ${ }^{1}$ However, alkali atoms and some alkaline earth atoms are well-known exceptions to this rule. ${ }^{2-7}$ For these dopants, the relatively diffuse valence $s$ electron produces a strong Pauli repulsion when close to helium atoms that overwhelms the weak dispersion force. As demonstrated by spectroscopic techniques and confirmed by theoretical studies, the alkali atom sits in a dimple on the helium droplet surface.

Pauli repulsion is equally dominant for small alkali clusters, so these remain on the surface of the liquid helium. However, Stark and Kresin suggested that a sufficiently large alkali cluster will eventually favor an interior location because the price paid by creating an interior bubble of sufficient size is more than compensated for by the net attractive (dispersive) van der Waals interactions between the alkali cluster and the surrounding liquid helium. ${ }^{8}$ Using a classical model that accounted for van der Waals forces through a standard Lennard-Jones description, together with a simple treatment of the surface tension of the interior bubble, Stark and Kresin were able to predict the critical size $n$ required to submerge $M_{n}$ clusters, where $M=\mathrm{Li}, \mathrm{Na}, \mathrm{K}, \mathrm{Rb}$, and Cs.

Recently, we provided the first experimental evidence showing that an alkali cluster submerges within the helium once it reaches a sufficient size. ${ }^{9}$ This work, which focused on sodium clusters, employed electron impact mass spectrometry. By recording mass spectra as a function of electron energy, it was shown that small sodium clusters ionize mainly through Penning ionization. However the threshold ion yield curves changed for cluster sizes of $n \geqslant 21$, showing behavior more consistent with charge transfer from $\mathrm{He}^{+}$. Underlying these observations is the expectation that the excited states of helium atoms responsible for Penning ionization, $\mathrm{He} 2{ }^{3} \mathrm{~S}$ and other metastable excited electronic states of helium at somewhat higher energies (which we subsequently refer to as $\mathrm{He}^{*}$ ), have a favored location near the helium droplet surface. ${ }^{7,10,11}$ This location is determined by the need to avoid expending additional energy in creating an interior bubble in which the $\mathrm{He}^{*}$ will reside. ${ }^{12}$ In contrast, any $\mathrm{He}^{+}$ions formed by electron impact have an equilibrium location at the center of the droplet, because the solvation energy by the surrounding helium atoms is maximized here..$^{13-16}$ Thus, this competition between Penning and charge transfer ionization is determined by the dopant location, with Penning ionization strongly favored for a surface-bound species and charge transfer favored for a submerged species. In the case of $\mathrm{Na}_{n}$, the transition from a surface-bound to a submerged cluster identified experimentally is in excellent agreement with the prediction by Stark and Kresin, whose calculations suggested a critical size for submersion of $n=21 .^{8}$

We expand on the earlier study of sodium clusters by switching to potassium clusters. A much larger minimum cluster size is expected for submersion of $\mathrm{K}_{n}$ clusters compared with $\mathrm{Na}_{n}$ because of the stronger repulsive interaction of $\mathrm{K}$ atoms with $\mathrm{He}$, which arises because the $4 s$ electron on each $\mathrm{K}$ atom is even more diffuse than the $3 s$ electron on $\mathrm{Na}$. This therefore poses a significant experimental challenge, because well-resolved mass spectra are required for large mass-tocharge ratios, $m / z$. Furthermore, because of the large range of cluster sizes, the ion count rate in any given mass channel is low, and this is exacerbated by the use of relatively low electron impact energies, where the ionization cross section is small compared to that obtained at electron energies $(60-100 \mathrm{eV})$ typical in standard electron impact mass spectrometry. We therefore employed a high-resolution mass spectrometer with a high duty cycle to record these weak signals. Consistent with expectation, we find that larger clusters are required for potassium than for sodium to achieve submersion. The threshold for $\mathrm{K}_{n}$ is not as sharp as that for $\mathrm{Na}_{n}$, but a clear change in ionization behavior has nevertheless been seen for $n>65$. As discussed later, this threshold is in good agreement 
with the theoretical prediction by Stark and Kresin. ${ }^{8}$ We also report observations of magic number cluster ions for both $\mathrm{K}_{n}{ }^{+}$ and $\mathrm{K}_{n}{ }^{2+}$ ions.

\section{EXPERIMENTAL DETAILS}

The experimental apparatus and procedure were recently described in detail, ${ }^{9}$ so only a brief account of key aspects is provided here. Neutral helium droplets were formed by supersonic expansion of ${ }^{4} \mathrm{He}(99.9999 \%$ purity) through a $5-\mu \mathrm{m}$ aperture in a platinum disk. This supersonic source was cooled to a temperature of $9.5 \mathrm{~K}$ and operated at a stagnation pressure of 20 bar. Under these conditions, we estimate that the mean helium droplet size is in the region of $10^{5}$ helium atoms.

The helium droplets are then skimmed and enter a differentially pumped vacuum chamber, where dopant pickup takes place. The pickup region is $6 \mathrm{~cm}$ in length, and potassium vapor was generated by oven evaporation, with the oven operated at a temperature of $130^{\circ} \mathrm{C}$. On leaving the pickup region, the nanodroplet beam is skimmed again and enters the differentially pumped ionization chamber. In this chamber, an electron beam of variable energy (0-150 eV) and current (10-200 $\mu \mathrm{A})$ crosses the helium droplet beam. The electron energy, which had a resolution of $\sim 1 \mathrm{eV}$, was varied between 11.5 and $27.5 \mathrm{eV}$ in $1 \mathrm{eV}$ steps to create positive ions. The ions are guided by a weak electrostatic field and accelerated toward the entrance zone of the time-of-flight mass spectrometer, arriving with a kinetic energy of $40 \mathrm{eV}$.

A commercial reflectron time-of-flight mass spectrometer (Tofwerk, model HTOF) with a mass resolution of $\sim 3500$ and a repetition rate of up to $\sim 8 \mathrm{kHz}$ was used. Typical acquisition times of $5 \mathrm{~h}$ were required to achieve adequate signal-to-noise ratios. After acquisition, the spectra were calibrated to a mass accuracy of better than 0.01 amu to account for any mass shifts occurring over the long data acquisition periods.

\section{RESULTS}

The basis for identifying the surface-to-interior transition is a difference in the ionization threshold behavior for clusters located in the interior of the helium droplets compared with those located on the surface. For surface-bound dopants, the ionization threshold should match the excitation energy required to form $\mathrm{He}^{*}(19.8 \mathrm{eV})$, whereas for dopants inside the droplet, the contribution from charge transfer via $\mathrm{He}^{+}$is more significant, skewing the threshold response toward the ionization energy of $\mathrm{He}(24.6 \mathrm{eV})$.

Figure 1 shows some selected ion yield curves as a function of incident electron energy for a range of cluster sizes. These curves represent the signal in the $\mathrm{K}_{n}{ }^{+}$channels, and the precise relationship that these might have to neutral $\mathrm{K}_{n}$ clusters are discussed later. In addition, these curves were normalized so that the relative signal intensities at $27.5 \mathrm{eV}$, the maximum shown in Fig. 1, are equal for all ions. The ion yield curves for $n \leqslant 70$ are similar (at least superficially), whereas those for $n=80$ and 90 are distinctly different. For the smaller clusters, there is a clear and relatively sharply rising ion signal well below the threshold for $\mathrm{He}^{+}$formation. The signal starts to rise above the baseline of $\sim 19.5 \mathrm{eV}$, which is consistent with

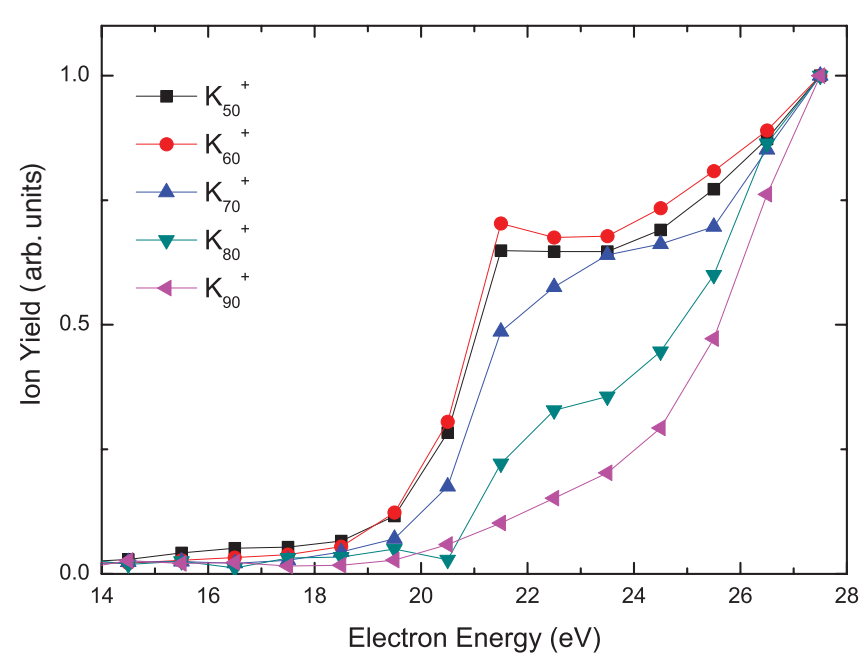

FIG. 1. (Color online) Ion yield curves as a function of electron energy for selected $\mathrm{K}_{n}{ }^{+}$cluster ions ( $n=50,60,70,80$, and 90). The curves were normalized such that the ion yields are the same at the maximum incident electron energy used $(27.5 \mathrm{eV})$.

the onset of Penning ionization. Above $\sim 21 \mathrm{eV}$, the signal seems to plateau to some extent and then begins to rise again, with a clear increase in slope $\sim 24 \mathrm{eV}$. This second rise is consistent with the start of ionization by $\mathrm{He}^{+}$. At $27.5 \mathrm{eV}$, the maximum electron energy shown in Fig. 1, the majority of ions for clusters with $n \leqslant 70$ are produced by Penning ionization.

For $n=80$ and 90, there is signal below an incident electron energy of $24 \mathrm{eV}$, suggesting a role for Penning ionization. However, the ion yield increases dramatically above $24 \mathrm{eV}$, and at $27.5 \mathrm{eV}$, the majority of the signal now comes from charge transfer via $\mathrm{He}^{+}$. Given that the primary dopant ionization channel is now charge transfer, this is consistent with the corresponding neutral clusters occupying an interior location in the helium droplets. In other words, assuming no significant ion fragmentation (described later), the data in Fig. 1 point to $\mathrm{K}_{n}$ cluster submersion beginning between $n=70$ and $n=80$.

An alternative way to display the data is to try to quantitatively account for the relative contributions from Penning ionization and charge transfer for specific $\mathrm{K}_{n}$ cluster sizes. To do this, we made the assumption that the ion yield curve for $\mathrm{K}_{2}{ }^{+}$derives almost entirely from Penning ionization (because $\mathrm{K}_{2}$ should reside at the droplet surface), whereas that from $\mathrm{K}_{90}{ }^{+}$arises entirely from charge transfer (assuming $\mathrm{K}_{90}$ is fully submerged). Specific functions can then be fitted to the ion yield curves, which can be superimposed for other cluster sizes. For $\mathrm{K}_{2}{ }^{+}$, we used the function

$$
f(E)=a+b\left(\frac{c}{1+10^{E-d}}+\frac{1-c}{1+10^{E-e}}\right)
$$

to represent the ion yield curve, where $E$ is the electron energy and $a, b, c, d$, and $e$ are adjustable parameters. For $\mathrm{K}_{90}{ }^{+}$, a simple exponential function was used to model the ion yield behavior. The quality of the fits for $\mathrm{K}_{2}{ }^{+}$and $\mathrm{K}_{90}{ }^{+}$is illustrated in Fig. 2. We then superimposed these two functions onto the ion yield data for other clusters sizes and used their relative amplitudes as adjustable parameters to achieve best agreement with the experimental results for other cluster sizes. As can 


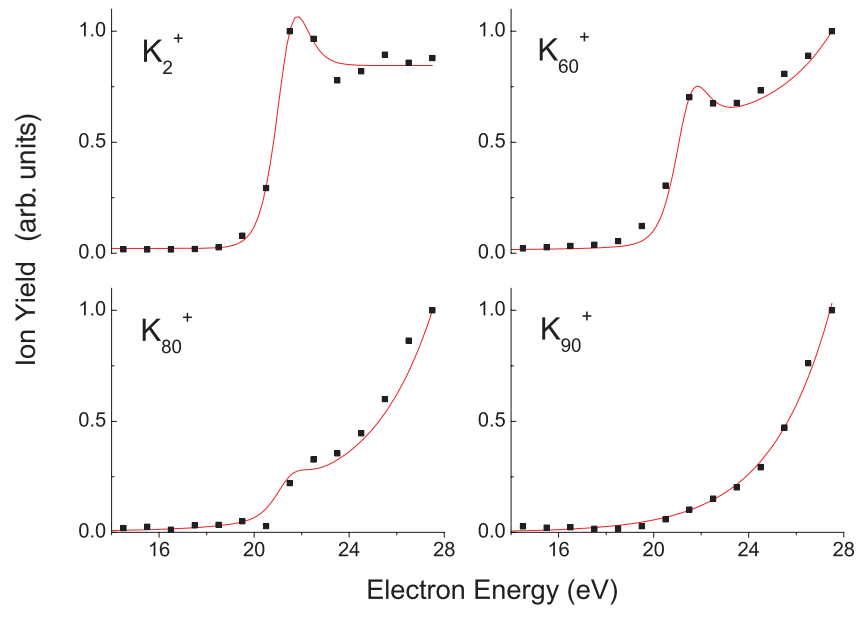

FIG. 2. (Color online) Comparison of ion yield curves for selected cluster ions. In the case of $\mathrm{K}_{2}{ }^{+}$, it is assumed that the dominant contribution derives from Penning ionization, whereas pure charge transfer ionization is assumed for $\mathrm{K}_{90}{ }^{+}$. Appropriate functions were chosen (see the main text for details) to fit the experimental data for $\mathrm{K}_{2}{ }^{+}$and $\mathrm{K}_{90}{ }^{+}$. Superpositions of these curves were then used for other ions, including $\mathrm{K}_{60}{ }^{+}$and $\mathrm{K}_{80}{ }^{+}$in this figure, to estimate the relative contributions of Penning and charge transfer ionization.

be seen in Fig. 2, this gives ion yield curves in reasonable agreement with experiment.

Figure 3 shows how the relative contributions of Penning and charge transfer ionization, derived from the preceding analysis, vary as a function of cluster size across the full range of clusters investigated. In Fig. 3, the ratio of contributions from Penning and charge transfer ionization is plotted on the vertical axis. This figure was also corrected to account for contributions from $\mathrm{K}_{n}{ }^{2+}$ cations (see the later discussion). This is easily done for $n \leqslant 30$, but for larger clusters this correction is not possible because the mass resolution is insufficient to distinguish monocation from dication contributions. Consequently, there is a discontinuity in Fig. 3, and we think it is likely that the Penning/charge transfer ratio is artificially lowered for $n>30$ because of hidden contributions from $\mathrm{K}_{n}{ }^{2+}$ ions, which give larger contributions to the charge transfer term than would otherwise be the case.

A marked decline in the Penning/charge transfer ratio, starting around roughly $n=65$ and continuing to beyond $n=80$, is evident in Fig. 3. This represents the transition zone from the surface location to the interior location for the $\mathrm{K}_{n}$ clusters.

\section{DISCUSSION}

\section{A. Surface-to-submersion transition}

The predicted submersion size from the calculations of Stark and Kresin is $n=78$ for $\mathrm{K}_{n}$ clusters. ${ }^{8}$ Because we identify full submersion around roughly $n=80$ from our experiments, the agreement between theory and experiment is excellent. We previously noted similarly impressive agreement with the Stark and Kresin model for $\mathrm{Na}_{n}$ clusters. ${ }^{9}$

However, potassium clusters show a distinct difference from sodium clusters. For the latter, there was a sharp change in ion yield behavior with cluster size, and the surface-interior

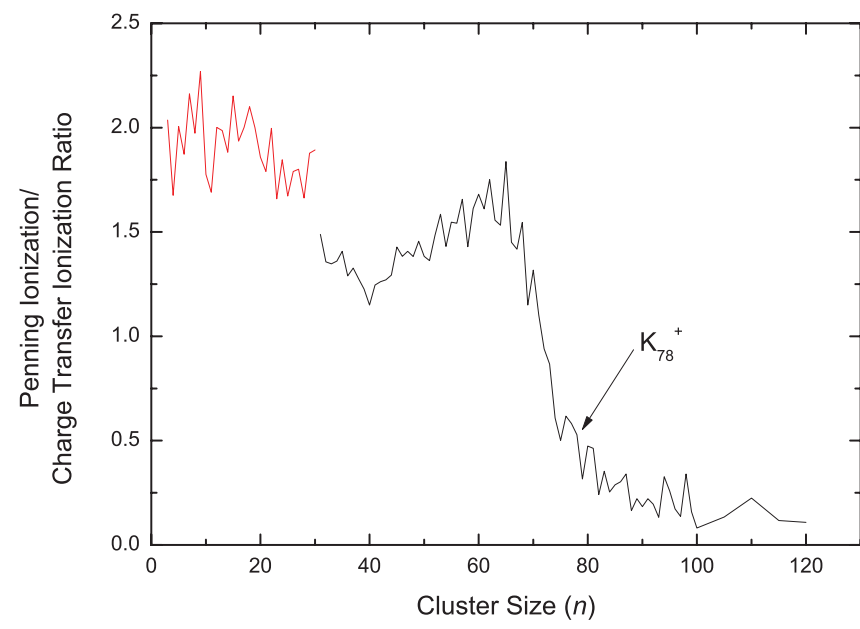

FIG. 3. (Color online) Relative contributions of Penning ionization versus charge transfer ionization plotted as a function of cluster size $n$. The data in the (red) line at the upper left were corrected to allow for dication contributions, but beyond $n=30$ this was not possible due to overlapping signal contributions from the monocations. $\mathrm{K}_{78}{ }^{+}$is highlighted because the corresponding neutral cluster is predicted to be the smallest $\mathrm{K}_{n}$ cluster that can submerge in liquid helium, according to calculations by Stark and Kresin (Ref. 8).

transition could be associated with a specific cluster ion, the $n=21$ species. For potassium, there is no such clarity, and the change from a surface to an interior location is more diffuse.

Ion fragmentation is not the source of this pronounced difference in surface-interior transition behavior for sodium and potassium clusters. Ion fragmentation undoubtedly occurs in our experiments, as evidenced by the observation of magic number features in the mass spectra (see the next section). Charge transfer is likely to be aggressive, with nearly $20 \mathrm{eV}$ of excess energy being available in charge transfer from $\mathrm{He}^{+}$ to $\mathrm{K}_{n}$. However, the fragmentation probability is unlikely to change significantly over the range $n=65-80$, not least because there is no structural shell closure expected in this region (as explained later). On the other hand, Penning ionization should be relatively soft, since the departing electron can remove most of the excess energy delivered by $\mathrm{He}^{*}$. Because Penning ionization is clearly the dominant contributor to the fall in the ratio of Penning/charge transfer probabilities in the transition zone, and because an almost one-to-one correspondence between the cation and the neutral cluster of a given size is expected for Penning ionization, the cluster sizes in the transition zone seen in Fig. 3 should closely reflect the neutral cluster sizes.

The rather gradual transition to full submersion is explicable in terms of the balance of forces at play in the competition between surface and interior binding. The cluster can sink into the interior when the energetic gain from the attractive dispersion force outweighs the energy that has to be expended in bringing the cluster into "contact" with the helium (Pauli repulsion) and for creating a bubble in the interior of the liquid. The Pauli repulsion between $\mathrm{K}$ and $\mathrm{He}$ is more unfavorable than that between $\mathrm{Na}$ and $\mathrm{He}$, so a larger cluster size for 
immersion is expected in the case of $\mathrm{K}_{n}$. In the theoretical prediction of alkali cluster submersion by Stark and Kresin, ${ }^{8}$ the switch from a surface-bound to a submerged cluster is defined by a crossover in the calculated energies for these two possibilities as a function of $n$ (see Figure Fig. 3 of Ref. 8) such that the energy of the interior cluster is now lower than that of the surface-bound cluster. The gradient of this energy versus $n$ plot for an interior cluster is much smaller for $\mathrm{K}_{n}$ than for $\mathrm{Na}_{n}$; therefore, the energies for clusters either side of the precise crossing point are similar over a reasonably large range of $n$. In other words, with a relatively large cluster, on the order of $n=70$, the addition or removal of a single $\mathrm{K}$ atom has only a small effect on the net dispersion energy and therefore may not be sufficiently decisive to force complete submersion.

From Fig. 3, we see that the surface-interior transition zone begins around $n=65$ and ends around roughly $n=80$. To understand what may be happening to the $\mathrm{K}_{n}$ clusters in this transition zone, we look at a possible analogy with $\mathrm{Mg}$ atoms. Until recently, there was considerable debate about the location of $\mathrm{Mg}$ atoms in/on helium droplets. Spectroscopic data, in the form of laser-induced fluorescence and resonance-enhanced multiphoton ionization measurements, ${ }^{17,18}$ seemed to point to an interior location, whereas mass spectrometry ion yield measurements, ${ }^{7}$ similar to those reported here, were consistent with a surface location (as found for all heavier alkaline earth metal atoms). These apparently contradictory findings were reconciled by density functional theory calculations, which show that although $\mathrm{Mg}$ becomes fully solvated in sufficiently large helium droplets ( $>200$ helium atoms), the energy lowering delivered by full solvation is relatively modest. ${ }^{19}$ As a consequence, $\mathrm{Mg}$ is highly delocalized within the helium droplet and therefore has the opportunity to occasionally explore the subsurface region. This dual nature of the $\mathrm{Mg}$ atoms, in which they show both interior and near-surface behavior, accounts for the experimental findings. A similar explanation may apply for $\mathrm{K}_{n}$ clusters near $n=70$ : that is, because the difference in energy between a surface and an interior location is small, the clusters undergo excursions within the droplet. Therefore, a portion of their trajectory takes them near the surface. As $n$ increases, the potential energy for surface locations becomes increasingly unfavorable, with the consequence that the $\mathrm{K}_{n}$ clusters become increasingly confined to central regions of the helium droplet.

Another factor that may contribute to the gradual surfaceinterior transition is the presence of a variety of structural isomers. In their prediction of the submersion size for alkali clusters, Stark and Kresin assumed perfect spherical shapes for the clusters. ${ }^{8}$ However, it is highly unlikely that a single structural arrangement will be adopted for all $\mathrm{K}_{n}$ clusters for a given value of $n$, particularly when $n>60$. Much more likely is a variety of structures, with some that are perhaps nearly spherical while others may be substantially anisotropic. The distinct cluster shapes will lead to distinct cluster submersion energetics, thus producing a blurred cluster submersion size. The role of structural isomers will be more important for potassium clusters than for sodium clusters, since the submersion into the helium occurs at much smaller cluster sizes for the latter. This may also help to explain why a sharp surface-interior transition is seen for $\mathrm{Na}_{n}$ but not for $\mathrm{K}_{n}$.

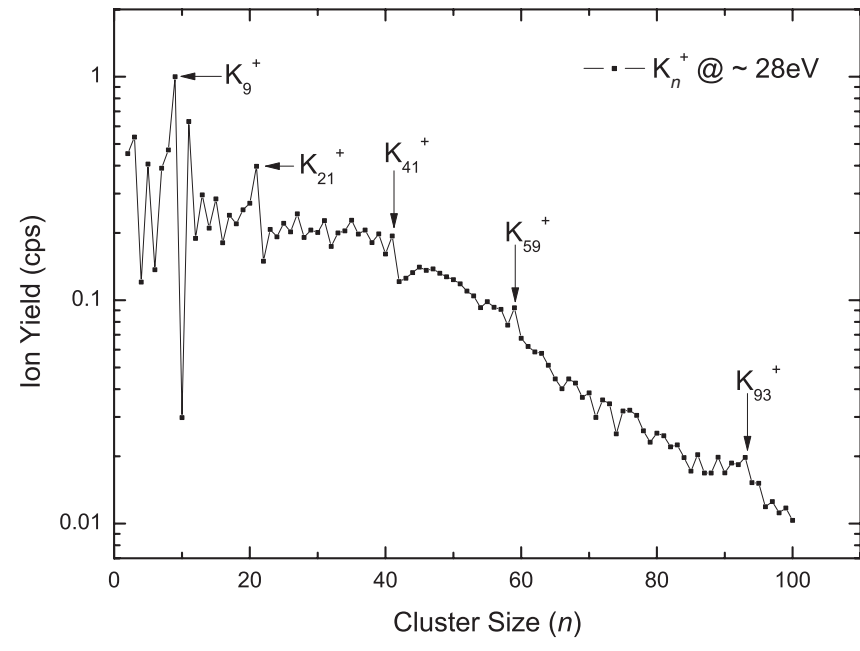

FIG. 4. Ion yield as a function of cluster size $n$ for $\mathrm{K}_{n}{ }^{+}$. Specific magic number features are labeled in the plot.

\section{B. $\mathrm{K}_{n}{ }^{+}$cluster ions: magic numbers}

Figure 4 shows the ion yield as a function of cluster size across the full range of cluster sizes studied. There are strong odd-even oscillations in abundance for small cluster sizes. Furthermore, prominent peaks are followed by a sharp drop in ion abundance for larger cluster ions, which is consistent with "magic number" species.

Our findings concur with work by Schulz et al., ${ }^{20}$ who investigated potassium cluster ions $(n \leqslant 25)$ produced by femtosecond photoionization of potassium clusters on helium nanodroplets. Odd-even intensity alternations were observed, along with pronounced maxima at $n=9$ and 21. These observations are consistent with the jellium model, which predicts enhanced stabilities for certain cluster sizes that lead to closed electronic subshells. ${ }^{21}$ In our work, we observed much larger potassium clusters than those seen by Schulz et al.; so, in addition to $\mathrm{K}_{9}{ }^{+}$and $\mathrm{K}_{21}{ }^{+}$, we also see magic number features for $\mathrm{K}_{41}{ }^{+}, \mathrm{K}_{59}{ }^{+}$, and $\mathrm{K}_{93}{ }^{+}$. All of these magic number cluster ions are expected from the jellium model. Magic numbers in mass spectra are formed when cluster ions with excess energy undergo consecutive fragmentation steps until they encounter a kinetic bottleneck, which is brought about by the enhanced stability of a particular cluster species. If the liquid helium was able to rapidly cool the ions before decomposition could take place, then no magic number ions would be observed and the ion cluster size distribution would be a perfect reflection of the neutral cluster size distribution. But magic numbers are observed in practice, suggesting inefficient cooling by the liquid helium in the current work, leading to at least some potassium clusters being released into the gas phase as relatively hot ions that then cool by sequential fragmentation.

\section{Potassium dications}

In addition to $\mathrm{K}_{n}{ }^{+}$ions, much smaller quantities of dications, $\mathrm{K}_{n}{ }^{2+}$, were detected. Figure 5 shows the ion yields for the dications as a function of cluster size. As with the monocations, a marked odd-even oscillation in abundances is observed, but in contrast to the monocations, it is the even-cluster ions that show the higher intensities relative to the 


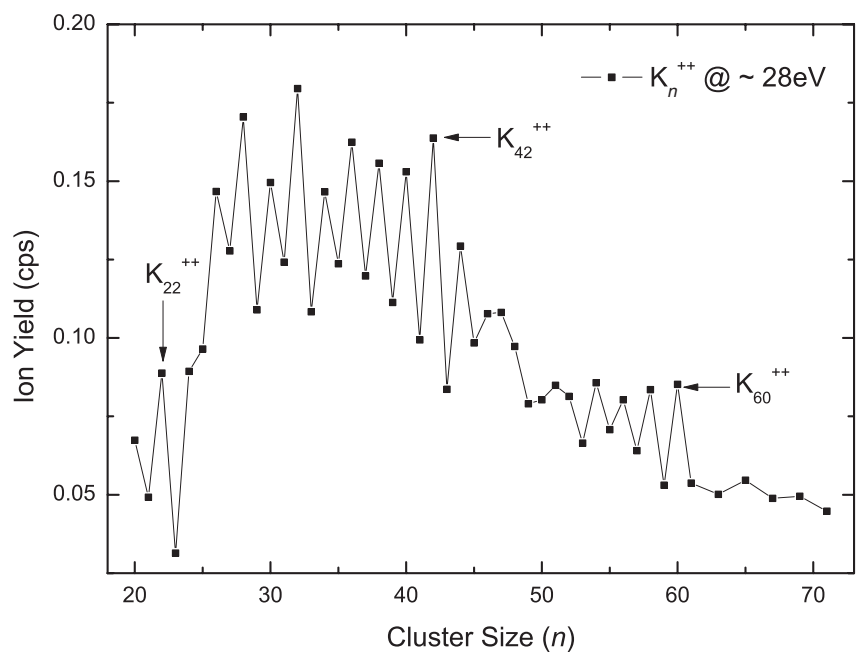

FIG. 5. Ion yield as a function of cluster size $n$ for $\mathrm{K}_{n}{ }^{2+}$. Possible magic number ions are identified in the plot.

odd-numbered dications. This, and the observation of tentative magic numbers for $\mathrm{K}_{42}{ }^{2+}$ and $\mathrm{K}_{60}{ }^{2+}$, are again consistent with the jellium model, since $\mathrm{K}_{n+1}{ }^{2+}$ delivers the same electronic structure as $\mathrm{K}_{n}{ }^{+}$in the jellium model.

Below a certain critical size, doubly charged cluster ions undergo Coulomb explosion to yield two singly charged fragment ions. For $\mathrm{K}_{n}{ }^{2+}$, the critical size has been determined to be $n_{c}=21 .^{22}$ The smallest dication observed from our experiments was $\mathrm{K}_{20}{ }^{2+}$, which is below the critical size limit. However, observation of this ion is plausible, because it has been shown that dications with $n>7$ are metastable due to the existence of a fission barrier. ${ }^{23}$ The barrier is clearly too low under our experimental conditions to allow sufficiently long survival of dications smaller than $\mathrm{K}_{20}{ }^{2+}$.

\section{CONCLUSIONS}

Experimental evidence has been presented showing that potassium clusters $\mathrm{K}_{n}$, once sufficiently large, submerge into helium nanodroplets. The transition from a surface-bound cluster to a fully solvated cluster in the interior of the helium droplet does not occur at a specific cluster size but instead appears to occur gradually over a range of cluster sizes. The midpoint of this transition is reasonably close to a recent theoretical prediction of $n=78$ for submersion into helium. The lack of a sharp surface-interior transition, in contrast to previous observations for $\mathrm{Na}_{n},{ }^{9}$ suggests that the location of $\mathrm{K}_{n}$ on the surface or inside the helium droplet is nearly isoenergetic over a significant range of $n$. Because $n$ needs to be much larger for $\mathrm{K}_{n}$ than for $\mathrm{Na}_{n}$ to defeat the more unfavorable Pauli repulsion, the addition or removal of a single $\mathrm{K}$ atom has a proportionately smaller effect on the solvation energetics for $\mathrm{K}_{n}$ than on those for $\mathrm{Na}_{n}$, thus blurring the surface-interior transition.

For the next member of the alkali group, Rb, theory suggests $\mathrm{Rb}_{n}$ submersion at $n=131,{ }^{8}$ but based on our experience with $\mathrm{K}_{n}$ clusters, we would expect an even more diffuse surfaceinterior transition for $\mathrm{Rb}_{n}$. Work is under way to see whether this transition is also observable.

\section{ACKNOWLEDGMENTS}

The work was supported by the Austrian Science Fund, Vienna (Contract No. P19073), and the European Commission, Brussels. A.M.E. is grateful to the University of Innsbruck for financial support. S.D. gratefully acknowledges the Austrian Academy of Sciences' Austrian Program for Advanced Research and Technology. L.A.d.L., P.B. and C.L. thank the University of Innsbruck for support via the Doktoratsstipendium aus der Nachwuchsförderung. *stephan.denifl@uibk.ac.at; andrew.ellis@le.ac.uk

${ }^{1}$ J. P. Toennies and A. F. Vilesov, Angew. Chemie Int. Ed. 43, 2622 (2004).

${ }^{2}$ F. Ancilotto, E. Cheng, M. W. Cole, and F. Toigo, Z. Phys. B 98, 323 (1995).

${ }^{3}$ F. Stienkemeier, J. Higgins, C. Callegari, S. I. Kanorsky, W. E. Ernst, and G. Scoles, Z. Phys. D 38, 253 (1996).

${ }^{4}$ J. H. Reho, C. Callegari, J. Higgins, W. E. Ernst, K. K. Lehmann, and G. Scoles, Faraday Discuss. 108, 161 (1997).

${ }^{5}$ A. Nakayama and K. Yamashita, J. Chem. Phys. 114, 780 (2001).

${ }^{6}$ J. Tiggesbäumker and F. Stienkemeier, PhysChemChemPhys. 9, 4748 (2007).

${ }^{7}$ Y. Ren and V. V. Kresin, Phys. Rev. A 76, 043204 (2007).

${ }^{8}$ C. Stark and V. V. Kresin, Phys. Rev. B 81, 085401 (2010).

${ }^{9}$ L. An der Lan, P. Bartl, C. Leidlmair, H. Schöbel, R. Jochum, S. Denifl, T. D. Märk, A. M. Ellis, and P. Scheier, J. Chem. Phys. 135, 044309 (2011).

${ }^{10}$ A. A. Scheidemann, V. V. Kresin, and H. Hess, J. Chem. Phys. 107, 2839 (1997).

${ }^{11}$ S. Vongehr and V. V. Kresin, J. Chem. Phys. 119, 11124 (2003).
${ }^{12}$ J. P. Toennies and A. F. Vilesov, Ann. Rev. Phys. Chem. 55, 1 (1998).

${ }^{13}$ A. Scheidemann, B. Schilling, and J. P. Toennies, J. Phys. Chem. 97, 2128 (1993).

${ }^{14}$ N. Halberstadt and K. C. Janda, Chem. Phys. Lett. 282, 409 (1998).

${ }^{15}$ W. K. Lewis, M. Lindsay, R. J. Bemish, and R. E. Miller, J. Am. Chem. Soc. 127, 7235 (2005).

${ }^{16}$ A. M. Ellis and S. Yang, Phys. Rev. A 76, 032714 (2007).

${ }^{17}$ J. Reho, U. Merker, M. R. Radcliff, K. K. Lehmann, and G. Scoles, J. Chem. Phys. 112, 8409 (2000).

${ }^{18}$ A. Przystawik, S. Göde, T. Döppner, J. Tiggesbäumker, and K.-H. Meiwes-Broer, Phys. Rev. A 78, 021202 (2008).

${ }^{19}$ A. Hernando, M. Barranco, R. Mayol, M. Pi, and F. Ancilotto, Phys. Rev. B 78, 184515 (2008).

${ }^{20}$ C. P. Schulz, P. Claas, D. Schumacher, and F. Stienkemeier, Phys. Rev. Lett. 92, 013401 (2004).

${ }^{21}$ W. D. Knight, K. Clemenger, W. A. de Heer, and W. A. Saunders, Phys. Rev. B 31, 2539 (1985).

${ }^{22}$ C. Bréchignac, Ph. Cahuzac, F. Carlier, and M. de Frutos, Nucl. Instrum. Meth. Res. B 88, 91 (1994).

${ }^{23}$ C. Bréchignac, Ph. Cahuzac, F. Carlier, M. de Frutos, R. N. Barnett, and U. Landman, Phys. Rev. Lett. 72, 1636 (1994). 